\title{
Aerobic exercise modulates cytokine profile and sleep quality in elderly
}

\author{
Shehab M Abd El-Kader ${ }^{1}$, Osama H Al-Jiffri ${ }^{2}$
}

1. Department of Physical Therapy, Faculty of Applied Medical Sciences, King Abdulaziz University, Jeddah, Saudi Arabia.

2. Department of Medical Laboratory Technology, Faculty of Applied Medical Sciences, King Abdulaziz University, Jeddah, Saudi Arabia.

\begin{abstract}
Background: Sleep disturbance is a major problem for older adults which can be exacerbated by increased inflammation as aging is associated with increased circulating pro-inflammatory and lower anti-inflammatory cytokines. There is a need to develop alternative medicine techniques to help improve sleep quality in the elderly.

Objective: To investigate the effects of aerobic exercise training on the sleep quality and inflammatory cytokines in elderly subjects.

Material and methods: Forty previously sedentary elderly subjects participated in this study, their age ranged from 61- 67 years. All subjects were randomly assigned to supervised aerobic exercise intervention group (group A, n=25) or control group (group $\mathrm{B}, \mathrm{n}=25)$. Polysomnographic recordings for sleep quality assessment, interleukin- 6 (IL- 6 ), tumor necrosis factor- alpha (TNF- $\alpha$ ) and interleukin-10 (IL-10) were measured before and after 6 months at the end of the study.

Results: There was a significant increase in total sleep duration, sleep efficiency and sleep onset latency in group(A) after 6 months of aerobic exercise training, while, wake time after sleep onset and rapid eye movement (REM) latency significantly reduced after 6 months of aerobic training compared with values obtained prior to aerobic exercise training. Also, the mean values of TNF- $\alpha$ and IL-6 decreased significantly and the mean value of IL-10 significantly increased in group (A) after the aerobic exercise training, however the results of the control group were not significant. Moreover, there were significant differences between both groups at the end of the study.

Conclusion: Exercise training can be considered as a non-pharmacological modality for modifying sleep quality and inflammation among elderly.

Keywords: Sleep quality, inflammatory cytokines, aerobic exercise, aging.

DOI: https://dx.doi.org/10.4314/ahs.v19i2.45

Cite as: Abd El-Kader SM, Al-Jiffri OH. Aerobic exercise modulates cytokine profile and sleep quality in elderly. Afri Health Sci.2019;19(2): 2198-2207. https://dx.doi.org/10.4314/abs.v19i2.45
\end{abstract}

\section{Introduction}

Aging sleep disturbance is a common problem reported by $39-75 \%$ of older persons ${ }^{1,2}$. Sleep disturbance may result in fatigue, emotional distress, difficulty with concentration and memory, daytime dysfunction and an increased

\section{Corresponding author: \\ Shehab M Abd El-Kader \\ Department of Physical Therapy, \\ Faculty of Applied Medical Sciences, \\ King Abdulaziz University, \\ P.O. Box 80324, Jeddah, 21589, Saudi Arabia. \\ Email: salmuzain@kau.edu.sa}

risk of falls; it has a negative impact on morbidity, mortality and quality of life $e^{3,4}$. However, sleep disturbance is not an inevitable part of aging ${ }^{3}$. The most frequent changes in sleep caused by aging are increases in the frequency nighttime awakenings and arousals and a decrease in the time spent in deep sleep ${ }^{5}$. Additional changes in sleep can also include increases in stages 1 and 2 sleep time, decreases in stages 3 and 4 sleep time, decreases in rapid eye movement (REM) sleep, increases in sleep fragmentation, decreases in total sleep time, decreases in sleep efficiency and increases in sleep disturbances ${ }^{5-7}$.

Because sleep complaints and sleep-related problems increase with age, it is possible that they are promoted by

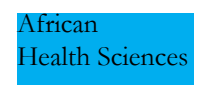

(C) 2019 Abd El-Kader et al. Licensee African Health Sciences. This is an Open Access article distributed under the terms of the Creative commons Attribution License (https://creativecommons.org/licenses/BY/4.0), which permits unrestricted use, distribution, and reproduction in any medium, provided the original work is properly cited. 
low-grade systemic inflammation present in older individuals $^{8,9}$. Indeed, this age-dependent pro-inflammatory state may be associated with several pathophysiological conditions associated with sleep complaints ${ }^{10}$, such as sleep disturbance ${ }^{11-13}$, in addition to many other age-related diseases, such as arteriosclerosis, dementia, osteoporosis, cancer ${ }^{14}$ and obesity ${ }^{15}$. Taken together, these factors can contribute to a poor quality of life for elderly individuals.

Because sleep complaints and sleep-related problems increase with age, it is possible that they are promoted by low-grade systemic inflammation present in older individuals ${ }^{16}$. Indeed, this age-dependent pro-inflammatory state may be associated with several pathophysiological conditions associated with sleep complaints ${ }^{17}$, such as sleep disturbance ${ }^{18,19}$

Sleep disturbance should be recognized and managed with either pharmacological or non-pharmacological treatment. Lower physical activity level was found to be a significant risk factor for insomnia ${ }^{20}$. Previous studies have reported that moderate-intensity exercise, such as Tai Chi or yoga may improve sleep quality ${ }^{21-23}$. In addition, participation in a regular exercise program can also have positive effects on sleep quality ${ }^{24}, \operatorname{mood}^{25}$, and $\operatorname{cog}$ nitive abilities ${ }^{26}$. A meta-analysis of 12 studies indicates that regular exercise increases total sleep time ${ }^{27}$. In addition, epidemiological data based on self-reports consistently support the view that acute and chronic exercise promotes sleep ${ }^{28}$.

Most studies examining the effects of exercise on sleep have focused on young good sleepers or fit athletes ${ }^{28,29}$. The limited data available in older adults also indicate an association between physical activity levels and sleep quality $^{30}$. There is also evidence that increasing physical activity level can improve sleep quality in older adults ${ }^{30,31}$. For example, in sedentary older adults, a 16 week program of mild to moderate intense physical activity improved self-rated sleep quality ${ }^{24}$. In another study, exposure to daily physical and social activity for just 2 weeks also had positive effects on sleep quality in older adults ${ }^{32}$. Furthermore, in older adults with depression, progressive weight training significantly improved measures of both subjective sleep quality and depression ${ }^{25}$. Moreover, moderate training may reduce resting plasma concentrations of pro-inflammatory cytokines and increase anti-inflammatory cytokines, consequently improving the quality of sleep $^{18}$.

African Health Sciences Vol 19 Issue 2, June, 2019
The aim of the study was to investigate the effect of aerobic exercise training on the sleep quality and inflammatory cytokines in elderly subjects.

\section{Patients and methods \\ Subjects}

Fifty healthy community dwelling sedentary men and women who were 61 years or older with symptoms of difficulty falling asleep and/or staying asleep, given theopportunity to sleep, accompanied by impairment in daytime functioning for at least 3 months. In addition, participants were required to be independent in activities of daily living and without significant cognitive deficits and have a sleep efficiency (SE) less than $80 \%$ and/or awaken earlier than desired if before $6 \mathrm{AM}$ and a total sleep time of less than 6.5 hours; Also, sedentary, defined as participation in exercise of mild to moderate intensity for less than 30 min per day and less than 2 times per week on a regular basis. Exclusion criteria included history of major psychiatric disorder as mania or alcohol or substance abuse or other neurological disorders, significant depressive symptoms, serious medical conditions or cardiopulmonary disease that contraindicate exercise, current use or use within the past month of psychoactive, hypnotic, stimulant or analgesic medications, shift work or other types of self-imposed irregular sleep schedules, smoking or caffeine consumption greater than $300 \mathrm{mg}$ per day. This study used a randomized parallel pre-post design. Participants were randomly assigned to either an aerobic exercise intervention group (group A) who participated in the exercise intervention conducted 3 times per week for 6 months or non-physical activity intervention and control group(group B) who received no training intervention and asked to follow their usual life style. This study was approved by the Ethical Committee for Scientific Research, Faculty of Applied Medical Sciences, King Abdulaziz University. All participants provided written informed consent.

\section{Measurements and procedures}

A. Sleep measures: All participants underwent polysomnographic (PSG) recording before and after the exercise training. For the pre-intervention assessment, PSG recording was performed over 2 nights. The first night served as an adaption night; 48 hour later, the participants returned for another night of PSG recording. The post-training PSG assessment occurred at least 30 hours after the last exercise session. The participants arrived at the sleep laboratory at 21:00; the PSG recording started 
and finished according to each volunteer's habitual sleep schedule. The room used for the recordings had a large comfortable bed, acoustic isolation, and controlled temperature and light. Recordings were conducted by a trained sleep technician using a digital system (Philips-Respironics, USA). The following parameters were analyzed: (a) total sleep time (in min), defined as the actual time spent asleep; (b) sleep latency (in min), defined as the time from lights out until the onset of three consecutive epochs of stage 1 or deeper sleep; (c) sleep efficiency, defined as the percentage of total recording time spent asleep; (d) wake after sleep onset (in min), defined as the total time scored as wakefulness between sleep onset and final awakening; (e) sleep stages 1, 2, 3, and 4 as well as REM sleep as percentages of total sleep time; and (f) latency to REM, defined as the time from sleep onset until the first epoch of REM sleep ${ }^{33}$.

B. Inflammatory cytokines assessment: Blood samples were collected from the antecubital vein after a 12hour fasting, the blood samples were centrifuged at +4 ${ }^{\circ} \mathrm{C}(1000=\mathrm{g}$ for $10 \mathrm{~min})$. Interleukin-6 (IL-6) and Interleukin-10 (IL-10) levels were analyzed by "Immulite 2000"immunassay analyzer (Siemens Healthcare Diagnostics, Deerfield, USA). However, tumor necrosis factor-alpha $(\mathrm{TNF}-\alpha)$ was measured by ELISA kits (ELX 50) in addition to ELISA microplate reader (ELX 808; BioTek Instruments, USA).
C. Aerobic exercise training program: Patients in group (A) were submitted to a $40 \mathrm{~min}$ aerobic session on a treadmill (the initial, 5-minute warm-up phase performed on the treadmill at a low load, each training session lasted 30 minutes and ended with 5-minute recovery and relaxation phase) either walking or running, based on heart rate, until the target heart rate was reached, according to American College of Sport Medicine guidelines. The program began with 10 min of stretching and was conducted using the maximal heart rate index (HRmax) estimated by: 220 -age. First 2 months $=60-70 \%$ of HRmax, second 4 months $=70-80 \%$ of HRmax 34 .

\section{Statistical analysis}

The mean values of the investigated parameters obtained before and after six months in both groups were compared using paired " $t$ " test. Independent " $t$ " test was used for the comparison between the two groups $(\mathrm{P}<0.05)$.

\section{Results}

The two groups were considered homogeneous regarding the demographic variables. The mean age of the group (A) was $64.98 \pm 4.15 y$ years, and the mean age of group (B) was $65.72 \pm 3.86 y$ years. There was no significant differences in age, weight, height, body mass index (BMI), waist circumference, hip circumference, systolic blood pressure and diastolic blood pressurebetween both groups (figure 1).

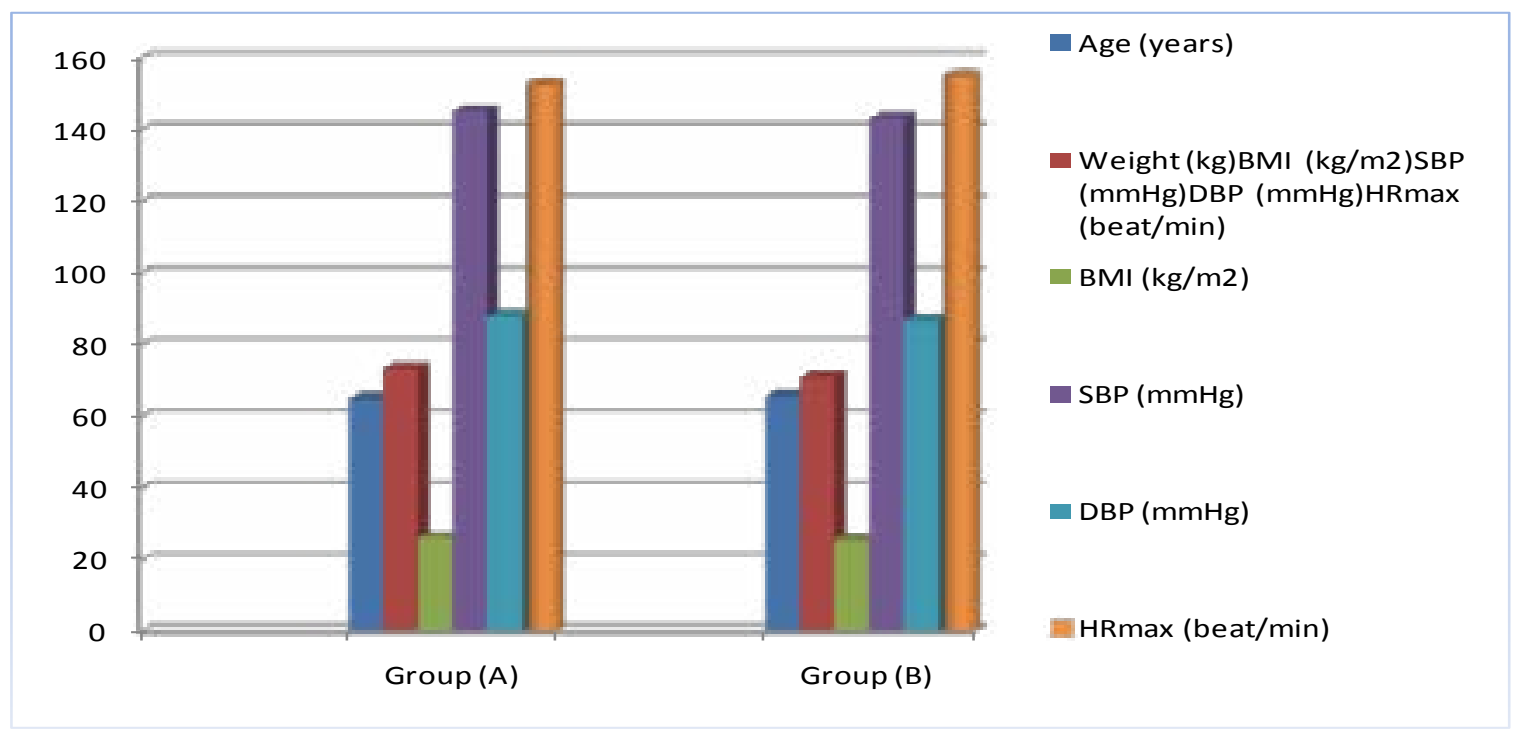

\section{Figure (1): Baseline characteristics of study participants.}


Regarding sleep quality parameter, there was a significantincrease in the total sleep duration, Sleep efficiency and Sleep onset latency in group(A) after 6 months of aero- bic exercise training, while, awake time after sleep onset and REM latency significantly reduced after 6 months of aerobic training compared with values obtained prior to aerobic exercise training (table 1 ).

Table 1: Mean value and significance of polysomnographic parameters, TNF$\alpha$, IL-6 and IL-10 in group (A) before and after treatment.

\begin{tabular}{|c|c|c|c|c|}
\hline & \multicolumn{2}{|c|}{ Mean + SD } & \multirow[t]{2}{*}{ t- value } & \multirow[t]{2}{*}{ Significance } \\
\hline & Before & After & & \\
\hline Total sleep duration (min) & $320.27 \pm 26.14$ & $336.81 \pm 29.25^{*}$ & 8.24 & 0.006 \\
\hline Sleep efficiency (\%) & $72.31 \pm 10.28$ & $81.24 \pm 11.47^{*}$ & 7.13 & 0.002 \\
\hline Sleep onset latency (min) & $12.85 \pm 3.16$ & $15.12 \pm 3.84^{*}$ & 6.91 & 0.018 \\
\hline Awake time after sleep onset (min) & $77.56 \pm 12.49$ & $63.44 \pm 10.23 *$ & 7.18 & 0.003 \\
\hline REM sleep latency (min) & $80.26 \pm 13.41$ & $65.12 \pm 11.37^{*}$ & 7.64 & 0.001 \\
\hline TNF- $\boldsymbol{\alpha}(\mathrm{pg} / \mathrm{mL})$ & $5.14 \pm 1.48$ & $3.25 \pm 1.21 *$ & 5.83 & 0.016 \\
\hline IL-6 (pg/mL) & $2.73 \pm 0.97$ & $1.64 \pm 0.75^{*}$ & 4.39 & 0.019 \\
\hline IL-10 (pg/ml) & $6.12 \pm 1.25$ & $8.34 \pm 1.63 *$ & 6.17 & 0.004 \\
\hline
\end{tabular}

REM: rapid eye movements; TNF- $\alpha$ : tumor necrosis factor - alpha; IL-6: Interleukin-6; IL-10: Interleukin$10 ;\left(^{*}\right)$ indicates a significant difference between the two groups, $\mathrm{P}<0.05$.

Moreover, the mean values of TNF- $\alpha$ and IL-6 decreased significantly and the mean value of IL-10 significantly increased in group (A) after the aerobic exercise training, however the results of the control group were not significant (table 2). Also, there were significant differences between both groups at the end of the study (table 3). 
Table 2: Mean value and significance of polysomnographic parameters, TNF- $\alpha$, IL- 6 and IL-10 in group (B) before and at the end of the study.

\begin{tabular}{|c|c|c|c|c|}
\hline & \multicolumn{2}{|c|}{ Mean + SD } & \multirow[t]{2}{*}{ t- value } & \multirow[t]{2}{*}{ Significance } \\
\hline & Before & After & & \\
\hline Total sleep duration (min) & $325.19 \pm 28.25$ & $318.65 \pm 28.72$ & 1.95 & 0.124 \\
\hline Sleep efficiency $(\%)$ & $74.23 \pm 12.46$ & $71.18 \pm 11.95$ & 1.64 & 0.061 \\
\hline Sleep onset latency $(\mathrm{min})$ & $13.92 \pm 4.15$ & $12.74 \pm 3.88$ & 1.21 & 0.152 \\
\hline Awake time after sleep onset (min) & $75.14 \pm 11.52$ & $77.28 \pm 11.49$ & 1.38 & 0.076 \\
\hline REM sleep latency (min) & $78.68 \pm 12.66$ & $79.56 \pm 12.41$ & 1.16 & 0.087 \\
\hline TNF- $\boldsymbol{\alpha}(\mathrm{pg} / \mathrm{mL})$ & $4.71 \pm 1.53$ & $4.95 \pm 1.62$ & 0.87 & 0.319 \\
\hline IL-6 (pg/mL) & $2.57 \pm 0.91$ & $2.82 \pm 0.93$ & 0.84 & 0.173 \\
\hline IL-10 (pg/ml) & $6.44 \pm 1.37$ & $6.27 \pm 1.35$ & 0.92 & 0.265 \\
\hline
\end{tabular}

REM: rapid eye movements; TNF- $\alpha$ : tumor necrosis factor - alpha; IL-6: Interleukin-6; IL-10: Interleukin-10.

Table 3: Mean value and significance of polysomnographic parameters, TNF- $\alpha$, IL- 6 and IL-10 in group (A) and group (B) at the end of the study.

\begin{tabular}{|c|c|c|c|c|}
\hline \multirow{2}{*}{ Total sleep duration (min) } & \multicolumn{2}{|c|}{ Mean + SD } & \multirow{2}{*}{ t- value } & Significance \\
\cline { 2 - 5 } & Group (A) & Group (B) & & 0.004 \\
\hline Sleep efficiency (\%) & $336.81 \pm 29.25^{*}$ & $318.65 \pm 28.72$ & 9.13 & 0.006 \\
\hline Sleep onset latency (min) & $81.24 \pm 11.47^{*}$ & $71.18 \pm 11.95$ & 7.86 & 0.012 \\
\hline Awake time after sleep onset (min) & $63.44 \pm 10.23^{*}$ & $77.28 \pm 11.49$ & 7.92 & 0.008 \\
\hline REM sleep latency (min) & $15.12 \pm 3.84^{*}$ & $12.74 \pm 3.88$ & 7.65 & 0.007 \\
\hline TNF-a (pg/mL) & $65.12 \pm 11.37^{*}$ & $79.56 \pm 12.41$ & 8.17 & 0.021 \\
\hline IL-6 (pg/mL) & $3.25 \pm 1.21^{*}$ & $4.95 \pm 1.62$ & 6.74 & 0.034 \\
\hline
\end{tabular}

REM: rapid eye movements; TNF- $\alpha$ : tumor necrosis factor - alpha; IL-6: Interleukin-6; IL-10: Interleukin-10; $\left({ }^{*}\right)$ indicates a significant difference between the two groups, $\mathrm{P}<0.05$. 


\section{Discussion}

Aging sleep disorder is an increasingly prevalent serious problem that affects more than $20 \%$ and $30 \%$, if older adults in western countries ${ }^{35}$. Lack of sleep can affect an individual's quality of life, social interaction and $\operatorname{mood}^{36}$. To date, the most common treatments for sleep disorders remain pharmacological agents as several systematic reviews have reported that hypnotics improve sleep quali$\mathrm{ty}^{37-39}$. However, the increased risk of adverse events was found to be statistically significant and poses potential risks for older individuals for falls or cognitive impairment ${ }^{39}$. Although research has found that pharmacological treatment appears an effective treatment for sleep disorders, evidence of its sustained efficacy is lacking ${ }^{40}$. Moreover, non-pharmacological approaches have a long history of treating sleep disorders, among which physical exercise is increasingly regarded as an effective way ${ }^{41,42}$. Concerning sleep quality parameter, there was a significant increase in the total sleep duration, sleep efficiency and sleep onset latency in group (A) after 6 months of aerobic exercise training, while, awake time after sleep onset and REM latency significantly reduced after 6 months of aerobic training compared with values obtained prior to aerobic exercise training (table 2), these results are in line with many previous studies ${ }^{43-53}$.

Reid et al. had Seventeen sedentary elderly subjects with insomnia who had 16 weeks of aerobic physical activity. The clearly stated that physical activity improved sleep quality on the global Pittsburgh Sleep Quality Index (PSQI) score, sleep latency, sleep duration, daytime dysfunction and sleep efficiency ${ }^{43}$. Where, Lira et al. conducted a study on fourteen male sedentary, healthy, elderly volunteers performed moderate training for 60 minutes/ day, 3 days/week for 24 week at a work rate equivalent to the ventilatory aerobic threshold. They proved that sleep parameters, awake time and REM sleep latency were decreased after 6 months exercise training in relation baseline values ${ }^{44}$. In addition, Yang and colleagues completed a systematic review with meta-analysis of six randomized trials and provided data on 305 participants (241 female). Each of the studies examined an exercise training program that consisted of either moderate intensity aerobic exercise or high intensity resistance exercise. The duration of most of the training programs was between 10 and 16 weeks. All of the studies used the self-reported Pittsburgh Sleep Quality Index to assess sleepquality.
Compared to the control group, the exercise group had significantly reduced sleep latency and medication use ${ }^{45}$. While, Chen and coworkers enrolled twenty-seven participants in 12 weeks of Baduanjin exercise training, they showed that overall sleep quality, subjective sleep quality, sleep latency, sleep duration, sleep efficiency, and daytime dysfunction significantly improved after 12 weeks of intervention ${ }^{46}$. In addition, Santos et al. had twenty-two male, sedentary, healthy, elderly volunteers performed moderate training for $60 \mathrm{~min} / \mathrm{day}, 3$ days/week for 24 week at a work rate equivalent to their ventilatory aerobic threshold, their findings suggest that aerobic exercise training increased aerobic capacity parameters, decreased REM latency and decreased time awake ${ }^{47}$. Moreover, Passos and colleagues concluded that a 4-month intervention of moderate aerobic exercise delivered to twenty-one sedentary participants with chronic primary insomnia had polysomnographic data significantly improvements following exercise training, where total sleep time, sleep efficiency and rapid eye movements significantly increased. In addition, sleep onset latency and wake time after sleep onset significantly decreased following exercise training ${ }^{48}$. Regarding the mechanism underlying the effect of exercise on sleep, although the mechanisms by which training can improve sleep quality are not well understood. It has been proposed that exercise training improves sleep quality through increasing energy consumption, endorphin secretion, or body temperature in a manner that facilitates sleep for recuperation of the body ${ }^{49-51}$. Moreover, moderate training may reduce resting plasma concentrations of pro-inflammatory cytokines and increase anti-inflammatory cytokines, consequently improving the quality of sleep $^{52,53}$.

Our results demonstrate that aerobic exercise training causes reduction in TNF- $\alpha$ and IL- 6 levels, in addition to increase in IL-10 level which suggests that exercise training can reduce inflammation in elderly individuals with more significant changes following aerobic exercise training. Regarding the aerobic exercise training, our results agreed with several studies have shown that aerobic exercise promotes the modulation of inflammation ${ }^{54-56}$. Several large cohort studies have found a relationship between self-reported physical activity levels and systemic markers of inflammation: higher levels of physical activity are coupled to lower levels of circulating inflammatory markers in elderly individuals ${ }^{57-59}$. While, Nicklas et al. 
showed that regular aerobic exercise training was efficient in lowering IL-6 levels even without weight loss ${ }^{60}$. However, Kohut et al. reported that 10-months of aerobic exercise significantly reduces serum inflammatory mediators in older adults ${ }^{61}$. In addition, Bote et al. demonstrated that 8-months (2 sessions/week, 60-min/session) of aquatic-based exercise training tempered neutrophil activation (chemotaxis) and decreased systemic levels of IL-8 and noradrenalin compared to controls ${ }^{62}$. In addition, Ploeger et al. who reported that moderate aerobic exercise training has been recommended as an anti-inflammatory therapy ${ }^{63}$. Where, Gatta and co-workers examined the expression of cytokines at both rest and following a bout of isokinetic exercise performed before and after 12 weeks of resistance exercise training in 8 young and 8 elderly. They stated that expression of pro-inflammatory cytokines MCP-1, IL-8 and IL-6 increased substantially after acute exercise. By contrast, the expression of the anti-inflammatory cytokines IL-4, IL-10 and IL-13 increased only slightly (or not at all) after acute exercise. These responses were not significantly different between young and elderly men, either before or after 12 weeks of exercise training. They concluded that, regular exercise training might help to normalize this inflammatory response in the elderly ${ }^{64}$. Moreover, Santos et al. stated the levels of IL- 6 and TNF- $\alpha$ and the ratio of TNF- $\alpha /$ IL-10 were decreased, whereas IL-10 levels were increased after 24 weeks moderate aerobic training. ${ }^{47}$

The three possible mechanisms of exercise anti-inflammatory effects include reduction in visceral fat mass65; reduction in the circulating numbers of pro-inflammatory monocytes ${ }^{66}$ and an increase in the circulating numbers of regulatory $\mathrm{T}$ cells ${ }^{67}$. Moreover, Hong and colleagues show that cardiorespiratory fitness is associated with reduced low grade inflammation that may in part be mediated by enhancing the ability of immune cells to suppress inflammatory responses via adrenergic receptors ${ }^{68}$. The training-induced sleep changes associated with an improvement in the inflammatory profile, demonstrated by a significant decrease in the level of inflammatory cytokines which were sufficient to improve global sleep phases as older individuals may demonstrate elevated inflammatory cytokines concentrations, which can increase non-rapid eye movement (NREM) sleep by impairing a subset of sleep-related neurons in the preoptic area/basal forebrain ${ }^{18,19,69}$.
The current study has important strengths and limitations. The major strength is the supervised nature of the study. All exercise sessions were supervised essentially $100 \%$. Moreover, the study was randomized; hence, we can extrapolate adherence to the general population. In the other hand, the major limitations is only elderly enrolled in the study, so the value of this study only related to this age group, also small sample size in both groups may limit the possibility of generalization of the findings in the present study. Finally, within the limit of this study, exercise training is recommended for improving sleep quality and modulation of low grade systemic inflammation among elderly. Further researches is needed to explore the impact of other therapeutic modalities on quality of sleep and other biochemical parameters among elderly.

\section{Conclusion}

Exercise training can be considered as a non-pharmacological modality for modifying sleep quality and inflammation among elderly.

\section{Acknowledgment}

This project was funded by the Deanship of Scientific Research (DSR), King Abdulaziz University, Jeddah, under grant no. (G-7-142-1439). The authors, therefore, acknowledge with thanks DSR technical and financial support.

\section{Conflict of interest}

None declared.

\section{References}

1. Blay SL, Baxter AS, Leite GF. Prevalence of self-reported sleep disturbance among older adults and the association of disturbed sleepwith service demand and medical conditions. International psychogeriatrics. 2008;20 (3):582-595.

2. Wong WS, Fielding R.Prevalence of insomnia among Chinese adults in Hong Kong: a population-based study. Journal of Sleep Research. 2011; 20:117-126.

3. Ancoli-IsraelS, Ayalon L, Salzman C. Sleep in the elderly: normal variations and common sleep disorders. Harvard Review of Psychiatry. 2008;16 (5): 279-286.

4. Ayalon L, Liu L, Ancoli-Israel S. Diagnosing and treating sleep disorders in the older adult. The Medical Clinics of North America. 2004;88 (3):737-750. 
5. Dam T'T, Ewing S, Ancoli-Israel S, Ensrud K, Redline $\mathrm{S}$, Stone K. Association between sleep and physical function in older men: the osteoporotic fractures in men sleep study. J Am Geriatr Soc. 2008;56:1665-73.

6. Sansoni P, Vescovini R, Fagnoni F, Biasini C, Zanni F, Zanlari L, et al. The immune system in extreme longevity. Exp Gerontol. 2008;43:61-5.

7. Chasens ER, Sereika SM, Weaver TE, Umlauf MG. Daytime sleepiness, exercise, and physical function in older adults. J Sleep Res. 2007;16:60-5.

8. Colbert LH, Visser M, Simonsick EM, Tracy RP, Newman AB, Kritchevsky SB, et al. Physical activity, exercise, and inflammatory markers in older adults: findings from the health, aging and body composition study. J Am Geriatr Soc. 2004;52:1098-104.

9. Murasko DM, Goonewardene IM. T-cell function in aging: mechanisms of decline. Annu Rev Gerontol Geriat 1990;10:71-96.

10. Gozal D. Sleep, sleep disorders and inflammation in children. Sleep Med. 2009;10(Suppl 1):S12-6.

11. Kapsimalis F, Basta M, Varouchakis G, Gourgoulianis K, Vgonzas A, Kryger M. Cytokines and pathological sleep. Sleep Med. 2008;9:603-14.

12. Krueger JM. The role of cytokines in sleep regulation. Curr Pharm Des. 2008;14:3408-16.

13. Santos RV, Tufik S, de Mello MT. Exercise, sleep and cytokines: is there a relation? Sleep Med Rev. 2007;11:2319.

14. Weinert BT, Timiras PS. Theories of aging. J Appl Physiol. 2003;95:1706-16.

15. Chandra RK. Nutrient regulation of immune functions. Forum Nutr. 2003;56:147-8.

16. Colbert LH, Visser M, Simonsick EM, Tracy RP, Newman AB, Kritchevsky SB, et al. Physical activity, exercise, and inflammatory markers in older adults: findings from the health, aging and body composition study. $\mathrm{J} \mathrm{Am}$ Geriatr Soc. 2004;52:1098-1104.

17. Gozal D. Sleep, sleep disorders and inflammation in children. Sleep Med. 2009;10 (Suppl 1): S12-S16

18. Kapsimalis F, Basta M, Varouchakis G, Gourgoulianis K, Vgonzas A, Kryger M. Cytokines and pathological sleep. Sleep Med. 2008;9:603-614.

19. Krueger JM. The role of cytokines in sleep regulation. Curr Pharm Des. 2008;14:3408-3416.

20. Morgan K. Daytime activity and risk factors for latelife insomnia. Journal of Sleep Research. 2003;12:231-238.

21. Chen KM, Chen MH, Chao HC, Hung HM, Lin HS, Li CH. Sleep quality, depression state, and health status

African Health Sciences Vol 19 Issue 2, June, 2019 of older adults after silver yoga exercises: cluster randomized trial. International Journal of Nursing Studies. 2009;46 (2):154-163.

22. Irwin MR, Olmstead R, Motivala SJ. Improving sleep quality in older adults with moderate sleep complaints: a randomized controlled trial of Tai Chi Chih Sleep. 2008; 31 (7):1001-1008.

23. King AC, Pruitt LA, Woo S, Castro CM, Ahn DK, Vitiello MV, Woodward SH, Bliwise DL. Effects of moderate-intensity exercise on polysomnographic and subjective sleep quality in older adults with mild to moderate sleep complaints. Journals of Gerontology Series A: Biological Sciences and Medical Sciences 2008;63 (9):997-1004.

24. King AC, Oman RF, Brassington GS, Bliwise DL, Haskell WL. Moderate-intensity exercise and self-rated quality of sleep in older adults. A randomized controlled trial. JAMA. 1997;277(1):32-7.

25. Singh NA, Clements KM, Fiatarone MA. A randomized controlled trial of the effect of exercise on sleep. Sleep. 1997;20(2):95-101.

26. Trinder J, Paxton SJ, Montgomery I, Fraser G. Endurance as opposed to power training: their effect on sleep. Psychophysiology. 1985;22(6):668-73.

27. Kubitz KA, Landers DM, Petruzzello SJ, Han M. The effects of acute and chronic exercise on sleep. A meta-analytic review. Sports Med. 1996;21(4):277-91.

28. Driver HS, Taylor SR. Exercise and sleep. Sleep Med Rev. 2000;4(4):387-402.

29. Youngstedt SD. Effects of exercise on sleep. Clin Sports Med. 2005;24(2):355-65 xi.

30. Edinger JD, Morey MC, Sullivan RJ, Higginbotham MB, Marsh GR, Dailey DS. Aerobic fitness, acute exercise and sleep in older men. Sleep. 1993;16(4):351-9.

31. King AC, Pruitt LA, Woo S, Castro CM, Ahn DK, Vitiello MV, et al. Effects of moderate-intensity exercise on polysomnographic and subjective sleep quality in older adults with mild to moderate sleep complaints. J Gerontol A Biol Sci Med Sci. 2008;63(9):997-1004.

32. Naylor E, Penev PD, Orbeta L, Janssen I, Ortiz R, Colecchia EF. Daily social and physical activity increases slow-wave sleep and daytime neuropsychological performance in the elderly. Sleep. 2000;23(1):87-95.

33. Rechtschaffen A, Kales AA. Manual of standardized terminology, techniques, and scoring system for sleep stages of human subjects. Los Angeles: Brain Information Service/Brain Research Institute/UCLA; 1968.

34. Robergs RA, Landwehr R. The surprising history of the "HRmax $=220$-age" equation. I ExercPhysiol Online. 2002; 5(2):1-10. 
35. Irwin MR, Cole JC, Nicassio PM. Comparative meta-analysis of behavioral interventions for insomnia and their efficacy in middleaged adults and in older adults 55+ years of age. Health Psychol. 2006;25 (1):3-14.

36. Jacobson B, Boolani A, Dunklee G, Shepardson A, Acharya H. Effect of prescribed sleep surfaces on back pain and sleep quality in patients diagnosed with low back and shoulder pain. Appl Ergon. 2010;42(1):91-97.

37. Nowell PM, Mazumdar S, Buysse DJ, Dew MA, Reynolds CF, Kupfer DJ. Benzodiazepines and zolpidem for chronic insomnia: a meta-analysis of treatment efficacy. JAMA.1997;278: 2170-2177.

38. Smith MT, Perlis ML, Park A, Smith MS, Pennington J, Giles DE. Comparative meta-analysis of pharmacotherapy and behavior therapy for persistent insomnia. American Journal of Psychiatry. 2002;159: 5-11.

39. Glass J, Lanctot KL, Herrmann N, Sproule BA, Busto UE. Sedative hypnotics in older people with insomnia: meta-analysis of risks and benefits. BMJ. 2005; 331: 1169 . 40. Morin CM, Hauri PJ, Espie CA, Spielman AJ, Buysse DJ, Bootzin RR. Nonpharmacologic treatment of chronic insomnia. An American Academy of Sleep Medicine review. Sleep. 1999;22:1134-1156.

41.Gooneratne NS. Complementary and alternative medicine for sleep disturbances in older adults. Clin Geriatr Med. 2008; 24 (1):121-138.

42.Yang PY, Ho KH, Chen HC, Chien MY. Exercise training improves sleep quality in middle-aged and older adults with sleep problems: a systematic review. J Physiother. 2012;58 (3): 157-163.

43. Reid KJ, Baron KG, Lu B, Naylor E, Wolfe L, Zee PC. Aerobic exercise improves self-reported sleep and quality of life in older adults with insomnia. Sleep Med. 2010;11(9):934-40.

44. Lira FS, Pimentel GD, Santos RV, Oyama LM, Damaso AR, Oller do Nascimento CM, Viana VA, Boscolo RA, Grassmann V, Santana MG, Esteves AM, Tufik S, de Mello MT.Exercise training improves sleep pattern and metabolic profile in elderly people in a time-dependent manner. Lipids Health Dis. 2011;6:10:1-6.

45. Yang PY, Ho KH, Chen HC, ChienMY.Exercise training improves sleep quality in middle-aged and older adults with sleep problems: a systematic review. J Physiother. 2012;58(3):157-63.

46. Chen M., Liu H, Huang H, Chiou A. The effect of a simple traditional exercise programme (Baduanjin exercise) on sleep quality of older adults: A randomized controlled trial. International Journal of Nursing Studies. 2012;49:265-273

47. Santos RV, Viana VA, Boscolo RA, Marques VG, Santana MG, Lira FS, Tufik S, de Mello MT. Moderate exercise training modulates cytokine profile and sleep in elderly people. Cytokine. 2012;60(3):731-5.

48. Passos GS, Poyares D, Santana MG, Teixeira AA, Lira FS, Youngstedt SD, dos Santos RV, Tufik S, de Mello MT. Exercise Improves Immune Function, Antidepressive Response, and Sleep Quality in Patients with Chronic Primary Insomnia. Biomed Res Int.2014;2014:498961.

49. Horne JA, Moore VJ . Sleep EEG effects of exercise with and without additional body cooling. Electroencephalography and Clinical Neurophysiology. 1985; 60: 33-38.

50. Driver HS, Taylor SR (2000) Exercise and sleep. Sleep Medicine Reviews. 4: 387-402.

51. Li F, Fisher KJ, Harmer P, Irbe D, Tearse RG, Weimer CJ . Tai chi and self-rated quality of sleep and daytime sleepiness in older adults: a randomized controlled trial. Journal of American Geriatrics Society. 2004;52: 892-900.

52. Yang PY, Ho KH, Chen HC, Chien MY. Exercise training improves sleep quality in middle-aged and older adults with sleep problems: a systematic review. J Physiother. 2012;58 (3): 157-163.

53. Kapsimalis F, Basta M, Varouchakis G, Gourgoulianis $\mathrm{K}$, Vgonzas A, Kryger M. Cytokines and pathological sleep. Sleep Med. 2008;9:603-14.

54. Donges CE, Duffield R, Drinkwater EJ. Effects of resistance or aerobic exercise training on interleukin-6, c-reactive protein, and body composition. Med Sci Sports Exerc. 2010;42:304-313.

55. Balducci S, Zanuso S, Nicolucci A, et al. Anti-inflammatory effect of exercise training in subjects with type 2 diabetes and the metabolic syndrome is dependent on exercise modalities and independent of weight loss. $\mathrm{Nu}$ trMetab Cardiovasc Dis. 2010;20:608-617.

56. Libardi CA, Souza GV, Cavaglieri CR, et al. Effect of resistance, endurance, and concurrent training on TNF-a, IL-6, and CRP. Med Sci Sports Exerc. 2012;44:50-56.

57. Geffken DF, Cushman M, Burke GL, Polak JF, Sakkinen PA, Tracy RP. Association between physical activity and markers of inflammation in a healthy elderly population. Am J Epidemiol 2001;153:242-250.

58. Colbert LH, Visser M, Simonsick EM, Tracy RP, Newman AB, Kritchevsky SB, Pahor M, Taaffe DR, Brach J, Rubin S, Harris TB. Physical activity, exercise, and inflammatory markers in older adults: findings from the Health, 
Aging and Body Composition Study. I Am Geriatr Soc. 2004;52:1098-1104.

59. Yu Z, Ye X, Wang J, Qi Q, Franco OH, Rennie KL, Pan A, Li H, Liu Y, Hu FB, Lin X. Associations of physical activity with inflammatory factors, adipocytokines, and metabolic syndrome in middle-aged and older Chinese people. Circulation. 2009;119:2969-2977.

60. Nicklas BJ, Hsu FC, Brinkley TJ, Church T, Goodpaster BH, Kritchevsky SB, Pahor M. Exercise training and plasma C-reactive protein and interleukin- 6 in elderly people. J Am Geriatr Soc. 2008;56:2045-2052.

61. Kohut ML, McCann DA, Russell DW, Konopka DN, Cunnick JE, Franke WD, Vanderah E. Aerobic exercise, but not flexibility/resistance exercise, reduces serum IL18, CRP, and IL-6 independent of beta-blockers, BMI, and psychosocial factors in older adults. Brain Behav Immun. 2006;20 (3): 201-209.

62. Bote ME, Garcia JJ, Hinchado MD, Ortega E. An exploratory study of the effect of regular aquatic exercise on the function of neutrophils from women with fibromyalgia: role of IL-8 and noradrenaline. Brain Behav. Brain BehavImmun 2014;39:107-12.

63. Ploeger HE, Takken T, de Greef MH, Timmons BW. The effects of acute and chronic exercise on inflammatory markers in children and adults with a chronic inflam- matory disease: a systematic review. Exerc Immunol Rev 2009;15:6-41.

64. Gatta P, Garnham A, Peake J, Cameron-Smith D. Effect of exercise training on skeletal muscle cytokine expression in the elderly. Brain, Behavior, and Immunity 2014;39: 80-86.

65. Mathur M, Pedersen B. Exercise as a mean to control low-grade inflammation. Mediators Inflamm 2008;2008:109502.

66. Timmerman K, Flynn M, Coen P, Markofski M, Pence B. Exercise training-induced lowering of inflammatory (CD14+CD16+) monocytes: a role in the anti-inflammatory influence of exercise? Leukoc Biol 2008; 84: 1271-1278.

67. Wang J, Song H, Tang X, Yang Y, Vieira VJ, Niu Y, $\mathrm{Ma} Y$. Effect of exercise training intensity on murine $\mathrm{T}$ regulatory cells and vaccination response. Scand J Med Sci Sports 2012; ;22(5):643-52.

68. Hong S, Dimitrov S, Pruitt C, Shaikh F, Beg N. Benefit of physical fitness against inflammation in obesity: role of beta adrenergic receptors. Brain Behav Immun. 2014;39:113-20.

69. Krueger JM, Fang J, Hansen MK, Zhang J, Obal-Jr F. Humoral regulation of sleep. News Physiol Sci 1998; 13:189-194. 